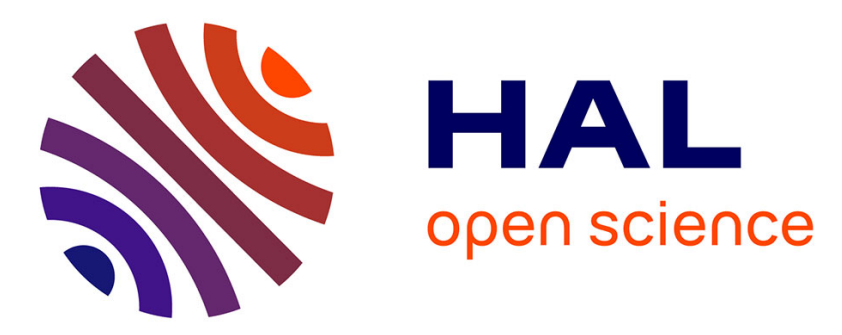

\title{
Investigating the Dynamic Instabilities of Model Granular Materials in Isotropic Consolidation and Triaxial Drained Compression
}

\author{
T. Nguyen, T. Doanh, A. Le Bot, D. Dalmas
}

\section{- To cite this version:}

T. Nguyen, T. Doanh, A. Le Bot, D. Dalmas. Investigating the Dynamic Instabilities of Model Granular Materials in Isotropic Consolidation and Triaxial Drained Compression. Sixth Biot Conference on Poromechanics, Jul 2017, Paris, France. 10.1061/9780784480779.223 . hal-01773675

\section{HAL Id: hal-01773675 \\ https://hal.science/hal-01773675}

Submitted on 27 Oct 2020

HAL is a multi-disciplinary open access archive for the deposit and dissemination of scientific research documents, whether they are published or not. The documents may come from teaching and research institutions in France or abroad, or from public or private research centers.
L'archive ouverte pluridisciplinaire HAL, est destinée au dépôt et à la diffusion de documents scientifiques de niveau recherche, publiés ou non, émanant des établissements d'enseignement et de recherche français ou étrangers, des laboratoires publics ou privés.

\section{(c)(1)}

Distributed under a Creative Commons Attribution| 4.0 International License 


\title{
Investigating the dynamic instabilities of model granular materials in isotropic consolidation and triaxial drained compression
}

\author{
T.T.T. Nguyễn ${ }^{1}, T$. Doanh ${ }^{1}$, A. Le Bot ${ }^{2}$ and D. Dalmas ${ }^{2}$ \\ ${ }^{1}$ Ecole Nationale des Travaux Publics de l'Etat. LGCB, LTDS (UMR 5513). 2 Rue M. \\ Audin, 69518 Vaulx en Velin Cedex. France. \\ ${ }^{2}$ Ecole Centrale de Lyon. LTDS (UMR 5513). 36 avenue Guy-de-Collongue, 69134 \\ Ecully Cedex. France.
}

\begin{abstract}
This paper reports the dynamic instabilities of model granular materials under isotropic consolidation and triaxial drained axisymmetric compression. Loose and fully saturated samples of mono sized glass beads exhibit in isotropic drained compression a series of local collapses under undetermined stress and even liquefaction in some rare cases. Stick-slip phenomenon occurs in drained compression, and even rare liquefaction happens for the first slip. These dynamic instabilities (collapse, liquefaction and stick-slip) of loose granular assembly can share the same physical driving mechanisms with strong links to geometrical features, i.e. the collapse of the structural metastable honeycombed macropores or the propagation of the local failures of the force chains; even if the unambiguous identification of the triggering mechanisms is still unknown. The experimental data eliminates the excess pore fluid as the primary cause.
\end{abstract}

\section{INTRODUCTION}

Despite a large number of intensive theoretical and experimental studies, the mechanical behaviour of granular materials is currently far from fully being understood $[10,2]$. One common idea is to use model granular materials consisting of perfectly rounded particles to overcome the complexity of real ones in laboratory as well as in discrete numerical modelling.

The stick-slip phenomenon in compression shear on glass beads was rapidly observed in geotechnical and geophysical laboratories [1, 3, 4, 5]; however, the precise triggering mechanisms were still missing. The isotropic drained compression is largely unstudied, leading to recent surprising discovery of isotropic liquefaction [6], the third kind of liquefaction in soil mechanics [7]. The unnecessary presence of external shearing stress for this liquefaction has thickened the mystery.

The aim of this paper is to explore some possible experimental links between these unexpected dynamic instabilities (collapse, liquefaction and stick-slip) of fully saturated model granular assembly. The temporal evolution of pore fluid pressure during the dynamic collapse and slip phase in triaxial testing is of particular interest. 


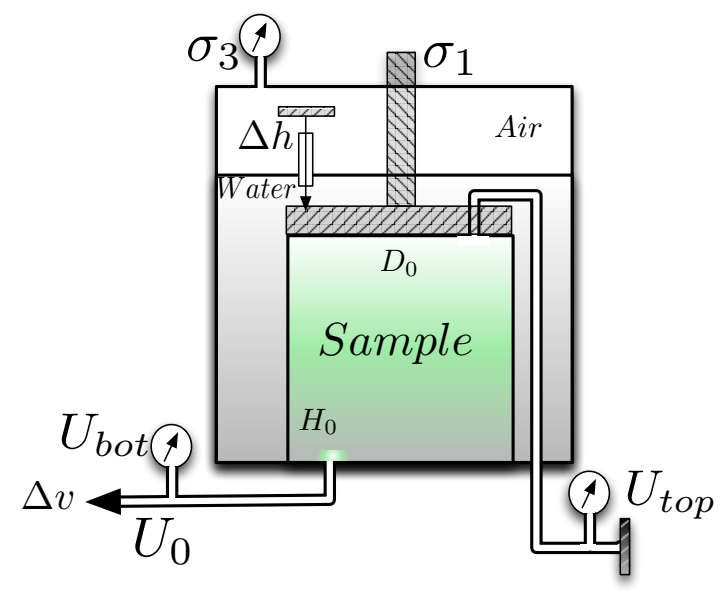

Figure 1. Experimental setup for isotropic consolidation and drained triaxial compression.

These links can contribute to a better understanding of the devastating liquefaction phenomenon during earthquake, still awaiting explanation.

\section{EXPERIMENTAL METHOD}

Measurement principle. In granular mechanics, the mechanical behaviour of fully saturated granular material is often studied in the versatile triaxial machine in two-step testing on a cylindrical sample. The isotropic drained compression (consolidation) is performed first, up to a desired effective isotropic stress $\sigma_{r}^{\prime}$ to get the compressibility characteristics; then the triaxial drained shear in compression to get the frictional angle and the dilatancy characteristics under constant $\sigma_{r}^{\prime}$. The drained character of these two steps is characterised by a nil interstitial pore pressure $U$ throughout the test duration, satisfying $\sigma^{\prime}=\sigma$, according to the effective stress principle $\sigma^{\prime}=\sigma+U, \sigma$ is the total stress [9]. These simple compressibility and shear characteristics are widely accepted to be the minimum ones in any theoretical constitutive equation on granular material.

Measurement setup. Fig. 1 presents the classical experimental set-up for triaxial testing on a short cylindrical specimen of initial height $H_{0}$ and diameter $D_{0}$ inside a triaxial cell. The granular assembly is enclosed inside a cylindrical and open-ended latex membrane of $0.3 \mathrm{~mm}$ thickness. The back pressure $U_{0}$ needed for a full saturation is applied at the bottom of the sample. The pore-water pressure $U$ is recorded on the top cap of the granular sample to ensure an homogeneous stress state.

Compressed air under constant stress rate is used in the first isotropic step up to a desired cell pressure $\sigma_{r}^{\prime}$. If the sample is still geometrically stable at $\sigma_{r}^{\prime}$, it will be vertically sheared under constant $\sigma_{r}^{\prime}$ and constant axial strain rate in the second shearing step. This second step adds an additional deviatoric shear stress $q=\sigma_{a}^{\prime}-\sigma_{r}^{\prime}$ to the first purely isotropic step; $\sigma_{a}^{\prime}$ is the axial stress. The excess pore pressure $\Delta U=$ 

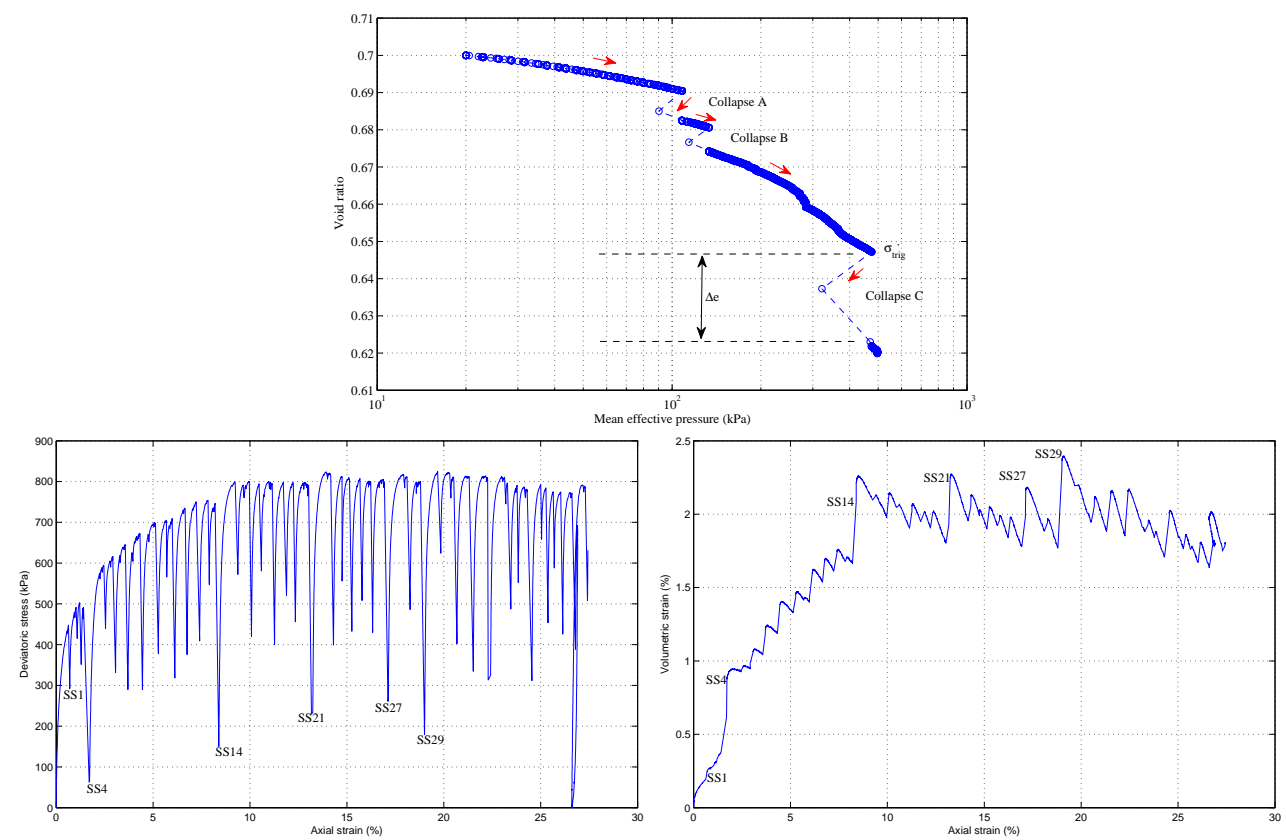

Figure 2. Collapses in isotropic consolidation from 20 to $500 \mathrm{kPa}$ (top) and stickslips in triaxial drained compression at $500 \mathrm{kPa}$ of confining pressure (bottom).

$U-U_{0}$ is kept small to respect the requirement of full drainage for these two steps (isotropic consolidation and drained compression). The global axial strain $\varepsilon_{a}=\Delta h / H_{0}$ is estimated with the measurement of axial displacement $\Delta h$ of the top platen inside the triaxial cell. The global volumetric strain is deduced from $\varepsilon_{v}=\Delta v / V_{0}$, where $V_{0}$ is the initial sample volume, with the measurement of water volume $\Delta v$ expelled from or moved into the sample [8]. The volumetric strain is usually translated into void ratio $e$ in the first isotropic step.

Materials and preparation. Virgin monodisperse and spherical soda-lime glass beads (CVP Sil-glass) of mean diameter of $0.723 \mathrm{~mm}$ is used. Granular sample, $70 \mathrm{~mm}$ in height $\left(H_{0}\right)$ and $70 \mathrm{~mm}$ in diameter $\left(D_{0}\right)$, is prepared using a modified moist tamping and under compaction method. The combination of water-soluble gas $\mathrm{CO}_{2}$ together with de-aired distilled water, and a high constant back pressure $U_{0}$ of $200 \mathrm{kPa}$ is used to ensure a quasi-saturated state through high Skempton's coefficient $\mathrm{B} \geq 0.95$.

\section{RESULTS AND ANALYSIS}

Isotropic consolidation. Fig. 2a shows the typical results of isotropic consolidation (the first part) with a very different compressibility behaviour. Instead of expected smooth and continuous increase of density with increasing $\sigma^{\prime}$, three events with large unexpected drops $\Delta e$ in void ratio can be seen at undetermined triggering isotropic stress $\sigma_{\text {trig. }}$. Between two events, continuous decrease of $e$ is observed. Event $\mathrm{C}$ with 


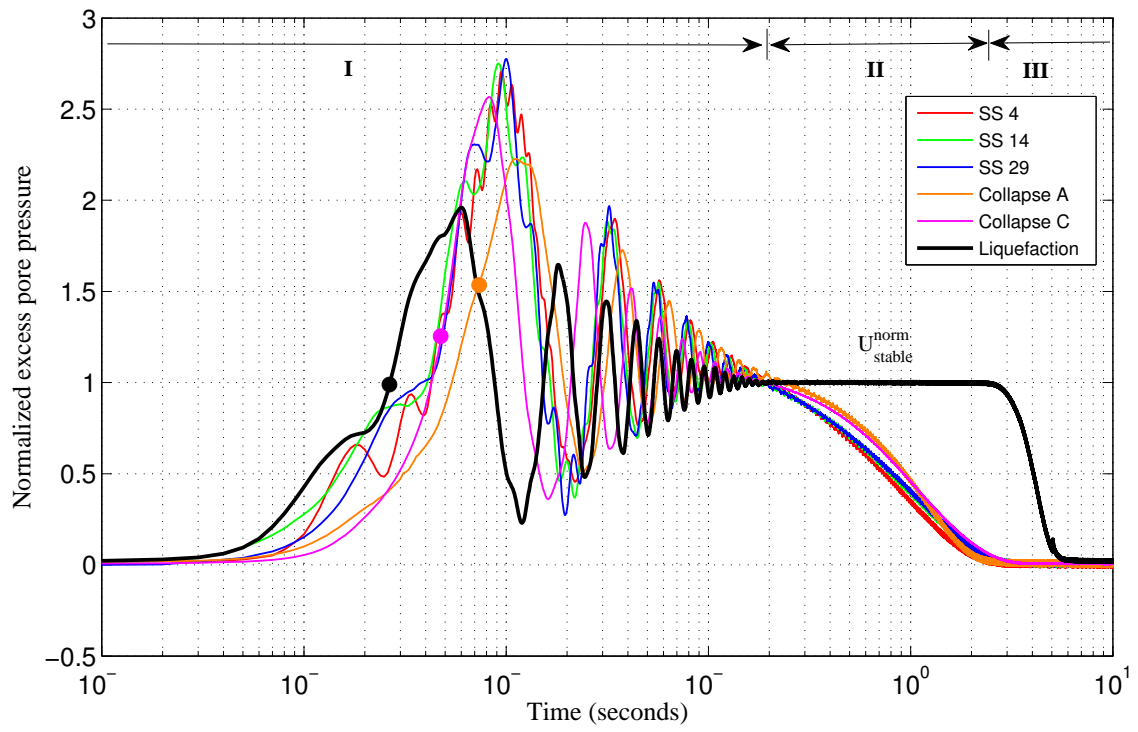

Figure 3. Three phases of pore water pressure development. Phase $I I$ is absent in isotropic collapses and compression stick-slips.

largest volumetric compaction occurs at $476 \mathrm{kPa}$, just before attaining the required 500 $\mathrm{kPa}$ of confining pressure of the drained triaxial compression (the second part). Small red arrows indicate the time direction.

Drained triaxial compression. Fig. $2 b$ and $c$ give the typical results of drained compression shear. Again, instead of expected smooth stress-strain behaviour and continuous increase of $q$ with increasing $\varepsilon_{a}$ for an initially loose granular material, stick-slip phenomenon was observed with numerous and quite irregular stick-slip events. Each event consists of a very abrupt deviatoric stress drop $\Delta q$ (slip-phase), followed by a gradual hardening (stick-phase) up approximatively to the previous stress level. Upon ignoring these stress drops, a nearly smooth envelope curve is retrieved, conforming to the usual behaviour of loose granular materials characterised by a monotonic increase of $q$ to a horizontal critical state plateau at $q^{\max }$. The stick-slips occur throughout the studied axial strain range, up to $25 \%$, with five particularly events of large deviatoric stress drop, noted as SS4 to SS29. Rapid succession of precursors with small stress drop can be seen, as before SS29. The largest drop towards the isotropic stress state at $27 \%$ of $\varepsilon_{a}$ is a simple unloading-reloading cycle with no stick-slip in unloading.

The corresponding volumetric strain exhibits strongly correlated variations with $\Delta q$ for the same values of $\varepsilon_{a}$. Globally, this volumetric strain confirms the loose character previously noticed on the stress-strain relationship with a classical continuous volumetric decrease towards its constant critical state at $2 \%$. The sawtooth volumetric profile seems to oscillate around this critical value. The slip-phase is always associated with a sudden volumetric compaction and the stick-phase a gradual dilation. Five large compactions are obviously related to the five large deviatoric stress drops. 

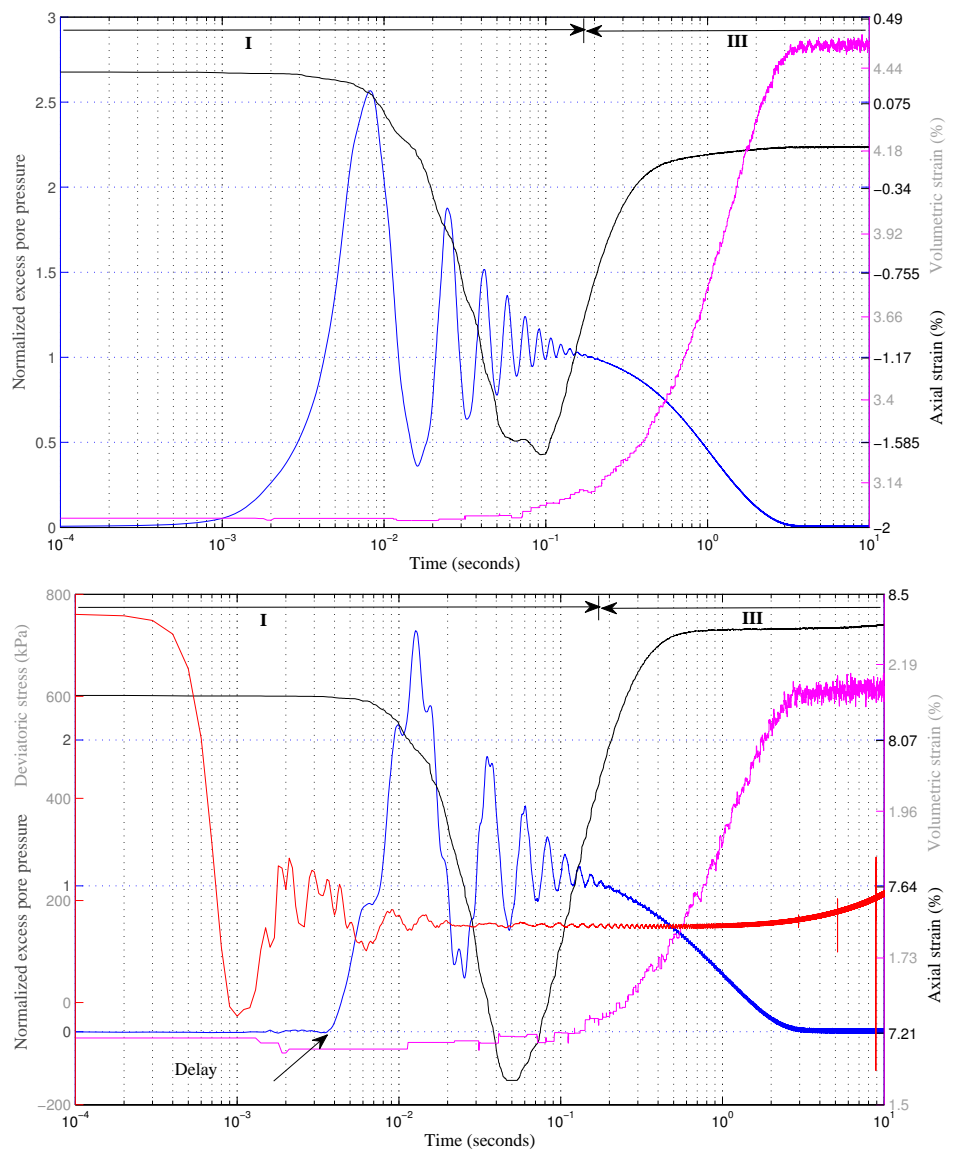

Figure 4. Temporal behaviour of isotropic collapse (top, collapse C) and compression stick-slip (bottom, stick-slip 14) with axial extension in transient phase: evolution of deviatoric stress, excess pore pressure, axial and volumetric strain.

Unexpected excess pore pressure. A closer look reveals that each event, isotropic (collapse and liquefaction) or deviatoric (stick-slip and liquefaction on first slip), commences with a sudden surge of $\Delta U$, followed by a rapid dissipation within a few seconds towards the equilibrium state of constant back-pressure $U_{0}$. It means an instantaneous reduction of effective stress $\sigma^{\prime}$, both axial and radial, according to the effective stress principle, followed by its gradual recover. It also means a very brief nonhomogenous stress state. In all events, the excess pore pressure in Fig. 3 vibrates like an oscillating underdamped system with a dominant frequency. $\Delta U$ was normalized by the brief stable value $\Delta U_{\text {stable }}$. Only selected events are shown to avoid clumsy figure. $\Delta U$ was measured simultaneously by two pore pressure transducers, one static (piezoresistive) and one dynamic (piezoelectric) with practically no differences.

The pore pressure evolution can be reasonably decomposed in three phases. The time origin is shifted to the beginning of the transient phase $\pm 0.1 \mathrm{~ms}$, which is the 


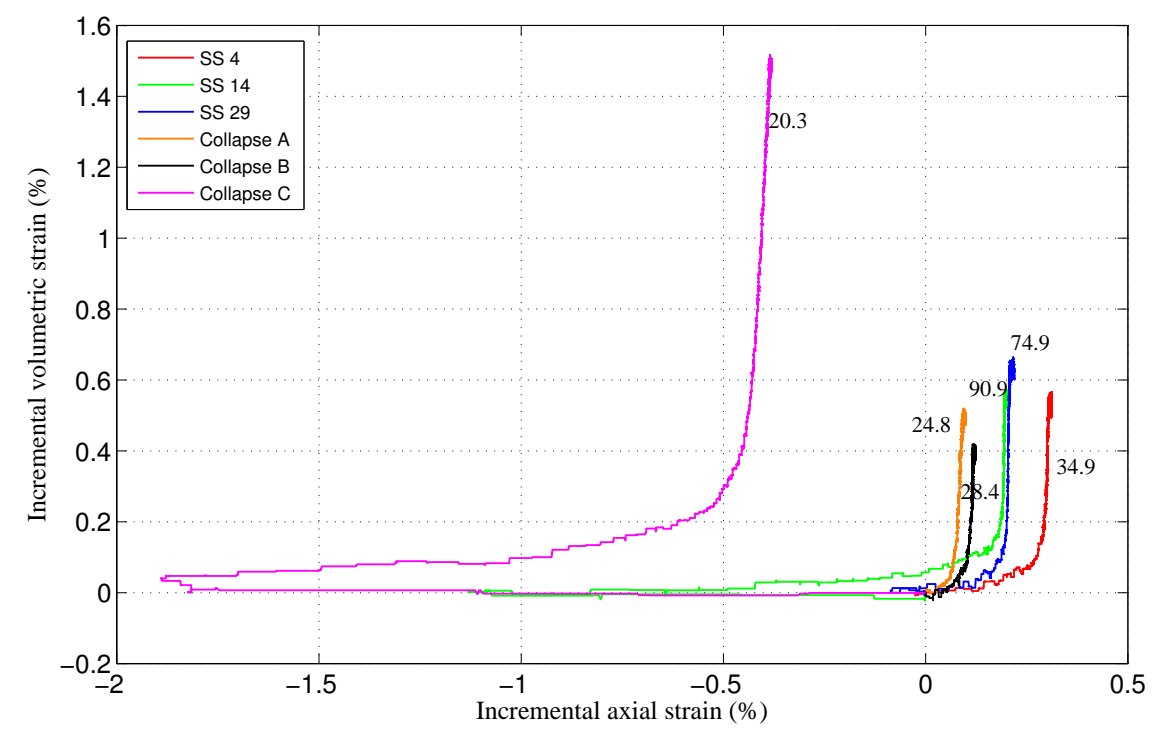

Figure 5. Dynamic incremental anisotropic coefficient $i_{d y n}$ in the transient phase.

current time resolution. First a fast transient phase $I$ occurred within $200 \mathrm{~ms}$ at nearly constant volume. Then appears a second phase $I I$ valid only for liquefaction event, characterized by a stabilized value of $\Delta U_{\text {stable }}$ for more than two seconds; and finally a last and longest dissipation phase $I I I$. The inferred liquefaction levels (solid circles) on the rising edge of $\Delta U$ of isotropic instabilities give the level of null effective stress. On liquefaction event it is exactly equal unity, meaning a null $\sigma^{\prime}$ for the whole phase $I I$, hence the terms liquefaction phase. Liquefaction event is obtained on separated sample.

Fig. 4 shows typical behaviour of isotropic collapses (event $\mathrm{C}$ ) and compression stick-slip (event SS14), grouping together the detailed development of $\Delta U, \varepsilon_{v}$ and $\varepsilon_{a}$. The deviatoric stress is added in case of stick-slip. The liquefaction case with very large axial and volumetric deformation is left aside to emphasize the most common instabilities with the notable absence of stabilised phase of $\Delta U$.

With the exception of sudden deviatoric stress drop $\Delta q$ to a constant level in less than 0.001 seconds, the temporal evolution of $\Delta U, \varepsilon_{v}$ and $\varepsilon_{a}$ during the slip phase are quite similar to those of spontaneous collapse under isotropic consolidation. Instead of usual jumps of $\varepsilon_{v}, \varepsilon_{a}$ and even $\Delta U$ in geotechnical and geophysical literature, this time-resolved study indicates a gradual dynamic modification of $\varepsilon_{v}$ and $\varepsilon_{a}$ from one to another steady level in less than 3 seconds, even less than 0.5 seconds for axial strain. Note the occurring of more than half of $\varepsilon_{a}$ during the fast transient phase $I$, the final contraction jump of $\varepsilon_{a}$ and the unexpected large extension state in between. These fast axial and volumetric strain rates suggest a dynamic regime for all instabilities.

Driving mechanisms. Fig. 4 also indicates one important insight into the dynamic slip. Since $\Delta U$ presents systematically an observed delay of about $3 \mathrm{~ms}$ comparing to the beginning of $\Delta q$, it cannot be the first cause of the slip instability. Data from stick- 
slip experiment are giving preliminary indications for plausible driving mechanisms of the observed instabilities. The brevity of $\Delta q$ strongly suggests a possible failure of contact force chains, and $\Delta U$ only as a consequence thereof. However, it still plays a significant contributor to the amplification of these dynamic instabilities of fully saturated media by reducing the effective stress. Dynamic instabilities still affect unsaturated and dry granular media, with lesser amplitude.

The dynamic incremental anisotropic coefficient $i_{d y n}=\Delta \varepsilon_{v} / \Delta \varepsilon_{a}$ and the spherical form of granular particles are highly suspected to be two major ingredients in creating these dynamic instabilities. Fig. 5 indicates the $i_{d y n}$ (black numbers) for selected events, estimated between the beginning and the end of each instability, once the excess pore pressure dissipated, regardless of the loading condition (isotropic or compression shear). Nearly parallel lines with constant $i_{d y n}$ in the dissipated phase $I I I$ are obtained with different mean values.

These experimental observations point to a plausible conjectural scenario in the structural instability of metastable microstructure within the granular assembly, supplemented by some experimental evidences of cascading collapses of the force chain. The sudden breakage or buckling of the preferential force chains can induce local dynamic shear deformation, and can result in a fast pore pressure buildup due to the incompressibility of water in a fully saturated media. The rolling feature of spherical grain shape can greatly facilitate these chain failures. Then the temporal decrease of the effective stress would promote further deformation. In some rare cases, it can lead to catastrophic liquefaction if the null effective stress can be maintained for a prolonged period of more than one second [6]. Unfortunately, the precise physical reasons are still unknown.

\section{CONCLUSIONS}

The mechanical behaviour of fully water-saturated monodisperse glass beads, initially assembled in loose state by moist-tamping technique is investigated. Drained isotropic compression and deviatoric shear are affected by dynamic instabilities: isotropic collapse and liquefaction, deviatoric stick-slip and possibly liquefaction on the first slip. Each event, regardless of the loading condition (isotropic or compression shear) and depositional methods, is characterised by three phases. The first dynamic transient phase $I$ has a fast drop of effective stress due to an excess pore pressure development which vibrates like an oscillating underdamped system. Local liquefaction with null $\sigma^{\prime}$ is attained. The second phase $I I$ of stabilised excess pore pressure can lead to liquefaction failure, if prolonged for more than one second. The quasi-static dissipated phase $I I I$ follows with gradual increase of axial and volumetric strain and a full recovery of $\sigma^{\prime}$ to the previous steady state before event.

These unexpected dynamic instabilities probably share the same underlying triggering mechanisms and the excess pore pressure is just the amplification factor on a fully saturated system, not the cause. The speculated structural instability of metastable microstructure is one of the plausible conjectural scenarios. However, the precise driv- 
ing mechanisms remain unknown.

\section{REFERENCES}

Adjemian, F., Evesque, P. (2004) Experimental study of stick-slip behaviour. Int. J. Num. Ana. Meth. in Geom., 28(6):501-530.

Andreotti, B., Forterre, Y., Pouliquen, O. (2013) Granular Media: Between Fluid and Solid. Cambridge University Press.

Anthony, J. L., Marone, C. (2005) Influence of particle characteristics on granular friction, J. Geophys. Res., 110, B08409.

Alshibli, K.A., Roussel, L.E. (2006) Experimental investigation of stick-slip behaviour in granular materials. Int. J. Num. Ana. Meth. in Geom., 30(14):1391-1407.

Doanh, T., Hoang, M.T., Roux, J.-N., Dequeker, C. (2013) Stick-slip behaviour of model granular materials in drained triaxial compression. Granular Matter, 15(1):1-23.

Doanh, T., Le Bot, A., Abdelmoula, N., Hans, S., Boutin, C. (2014) Liquefaction of immersed granular media under isotropic compression. Europhys. Lett., 108(2):24004.

Ishihara, K. (1996) Soil behaviour in earthquake geotechnics. Oxford University Press.

Lade, P.,V. (2016) Triaxial Testing of Soils. Wiley-Blackwell.

Terzaghi, K., Peck, R.P., Mesri, G. (1996) Soil Mechanics in Engineering Practice, 3rd Edition. Wiley.

Wood, D. M. (1990) Soil behaviour and critical state soil mechanics. Cambridge University Press. 